\title{
Use of probiotic, prebiotic and symbiotic in infant formulas.
}

\section{Federik M*}

Department of Collective Health, National University of Lanus, Argentina

\begin{abstract}
Introduction: Research and innovation in the field of infant milk formulas aim to approximate the nutritional composition of breast milk, and mimic its benefits at the functional level. Technological advances, together with the development of knowledge about the components of breast milk, have made it possible to identify a large number of bioactive compounds, which has led to their incorporation into infant formulas.

Methods: Bibliographic review of scientific publications, in the databases: SciELO, Scopus and ScienceDirect. The publications addressed the topics of probiotics, symbiotic and their addition in infant milk formulas.

Results: A total of 32 scientific articles were found that met the inclusion criteria. The definitions of prebiotic, probiotic and symbiotic are presented. The physiological effects on health and its recommendations are detailed.

Conclusions: Evidence about clinical efficacy through the addition of prebiotics is not enough to recommend routine use in infant formulas. In relation to the addition of probiotics, further research is needed to be able to establish with specificity the types of strains, species and doses of consumption that are beneficial for health.
\end{abstract}

Keywords: Prebiotics, Probiotics, Symbiotic, Infant formula.

Accepted on May 31, 2019

\section{Introduction}

Functional foods have their origins in Japan in the late 80 's. They emerged during the implementation of a government research program aimed at improving the health of children of school age. Through this program, the idea of enriching food arises with the purpose of knowing other functions of food, in addition to the main nutritive function [1,2]. From that moment a new range of foods was born called FOSHU (Food for Specific Health Use), which are characterized as natural or processed foods, with ingredients that play a specific benefit in one or more functions physiological aspects of the human organism beyond its nutritional content, and that, moreover, are part of the habitual diet $[3,4]$. This group of foods contributes in different functions of the organism, among them, it provides protection to the cardiovascular and gastrointestinal system, it allows the growth and optimal development, intervenes in the metabolism of substrates and the contribution of antioxidants, among others $[5,6]$. Currently, functional foods show a growing trend in the market. The foods that have been shown to have functional actions, aimed at children are few, among them are: probiotics (dairy fermented by Lactobacilli and Bifidobacteria), prebiotics, foods rich in omega-3 polyunsaturated fatty acids and foods with high content in fiber. However, its use presents scant scientific evidence supported by rigorous and validated studies in children [7-9].

In recent years the addition of functional ingredients in the composition of infant formula has evolved in order to achieve a nutritional content similar to that of breast milk. In this sense, it has been achieved that the content of macronutrients [carbohydrates, proteins and lipids] and micronutrients (vitamins and minerals) of infant formulas is similar to that of breast milk, being regulated by legally established standards [10]. However, due to its nutritional composition, breast milk contains a wide variety of essential compounds for infant feeding; among which are the bioactive compounds, responsible for a wide range of beneficial effects, such as the promotion of the maturation of the immune system, protection against infections, the modulation of metabolic pathways and the inflammatory response, among others.

The isolation and identification in human milk of oligosaccharides and bacteria with beneficial effects for the host, provides scientific support for the supplementation of infant formulas with these compounds, in order to mimic the functional effects observed in infants fed with breast milk.

Although breast milk is considered the best food option, approximately $47 \%$ of children in Argentina are fed formulas before 6 months of age [11]. Therefore, advances in scientific knowledge for the development and design of infant formulas are essential in the field of infant feeding. The objective of the research was to analyze in scientific journals, the publications that dealt with the issue of the addition of probiotics, prebiotics and symbiotic in infant formula. A non-systematic review of the scientific literature was carried out between March and May 2018. The objective was to analyze and describe the addition of probiotics, prebiotics and symbiotic in infant formulas, as well as the observed health effects in infants.

The articles analyzed were identified through the automated search in the SciELO, Scopus and ScienceDirect databases. The aforementioned bases were selected, considering that they 
provide sufficient breadth in the search around the subject that was investigated. The research was carried out using combinations of descriptors (Mesch Term) and free terms according to the base in which the search was carried out; these terms were: probiotics, prebiotics, symbiotic, infant and infant formula. In addition, the logical operators and/ or were used in order to recover the maximum possible quantity of scientific publications.

In addition to the exploration in the virtual libraries, a complementary search was carried out among the bibliographic references of the selected studies, in order to identify possible publications pertinent to the objective of the study. The initial search resulted in 69 found articles. After reading the full text, applying the inclusion and exclusion criteria, the documentary corpus was formed, which was made up of 32 articles. For the treatment of the information, a content analysis was carried out, identifying the units of meaning and units of registration. The variables considered for the investigation were: the type of prebiotics, probiotics and symbiotic used evidence on the effects they produce on health, dosage and regulation of them.

\section{Literature Review}

\section{Importance of adequate nutrition in the infant}

The first years of life are a critical moment, where proper nutrition is essential to maintain optimal growth and development of children [11]. During the first two years of life, food is a key factor, since all must be provided the macro and micronutrients necessary for the formation of organs, systems and structures of the body. During this period, nutritional deficiencies can leave sequels difficult to reverse $[12,13]$. In this sense, breast milk is the best food for infants, since it contains living cells that help fight different diseases, allows the maturation of all organs and tissues, and also prepares the body for the start of complementary feeding [14,15]. According to different research, exclusive breastfeeding has a protective effect against infections due to the contribution of immunoglobulins, presence of trace elements, prebiotics and immune cells, which raise the population of lymphocytes for the defense of the child, but not the same with the use of artificial formulas [14-20]. Currently there is a marked development in the chemical composition of infant formulas, thanks to the advancement of functional foods and the identification of bioactive components in breast milk. Situation that promoted the incorporation of these components in infant formula, with the objective of imitating the nutritional composition of breast milk and obtaining similar benefits [13].

\section{Use of functional foods in infant formulas}

Functional foods are defined as those foods where one of its components, whether or not it is nutrient, affects the functions of the organism in a specific and positive way. They must also promote a physiological or psychological effect, beyond their traditional nutritional value, which contributes to the maintenance of the state of health and the reduction of the risk of suffering from a certain disease [21]. A functional food can be a natural or modified food, or a combination of both. The functional ingredients can be classified according to the bioactive components present in the food in: prebiotics, probiotics, fibers, phytochemicals, vitamins, polyunsaturated fatty acids and certain peptides and proteins [22,23].

\section{Prebiotics}

Prebiotics are defined as nondigestible food ingredients, which selectively stimulate the growth or metabolic activity of a limited number of bacterial strains in the colon, and benefit the health of the host [24-26]. These compounds are characterized by being large molecules, resistant to digestion and hydrolysis in the upper part of the gastro digestive tract. Once they reach the large intestine, they are degraded by the bacterial microflora [27], mainly by Bifidobacterium and Lactobacilli, which reduces the number of putrefactive organisms and increases the saccharolytic species [28,29].

The prebiotics are considered oligosaccharides [OS], according to their chemical composition contain 3 to 30 molecules of monosaccharides linked by glycoside bonds. These include several types of OS [fructo-, galacto-, isomalto-, xylo- and soyoligosaccharides], lactulose and lactosucrose [26]. Only three non-digestible OSs present the characteristics to be considered and classified as prebiotics: the inulin that includes the fructooligosaccharides [FOS] (a type of inulin-fructans formed by short-chain oligomers], the galacto-oligosaccharides [GOS] ] [a type of galactans) and lactulose [24].

Prebiotics selectively stimulate the growth of Bifidobacteria and Lactobacilli, resulting in the production of growth inhibiting substances and pathogen survival. The partial fermentation of OS by bifidobacteria produces short chain fatty acids [FA], such as butyric, propionic, L-lactic and acetic acids. The first three favor the growth and differentiation of epithelial cells in vivo. Butyric acid is an important energy source for the colon. The acidification of the colonic environment inhibits the growth of bacteroides, clostridium and coliforms, and favors the production of mucin, which decreases bacterial colonization and translocation. Likewise, the intestinal microbiota associated with epithelial cells enhances the barrier effect of the epithelium, competing for nutrients and sites of attachment to the epithelium with pathogenic microorganisms.

The intestinal microbiota is able to control the expression of proteins (occludins and claudins) involved in regulating the intercellular space, as well as stimulating the production of antibacterial peptides known as defensins, and cytokines that help protect the host from pathogenic microorganisms [30,31].

Bacteria of the genus Bifidobacterium predominate in the intestinal microbiota of the infant exclusively fed with breast milk, compared to children fed infant formula, as a product of the high content of complex oligosaccharides formed by sialic acid, $\mathrm{N}$-acetylglucosamine, L-fucose, D-glucose and D-galactose. In breast milk the oligosaccharides are in a concentration of up to 12-14 $\mathrm{g} / \mathrm{L}$, constituting one of the main solid fractions of this food.

Currently the most commonly used prebiotics in infant feeding are fructooligosaccharides, galactooligosaccharides and inulin. Table 1 shows some examples of oligosaccharides and the type of available source.

The FOS used in most infant formulas is high molecular weight, derived from inulin, in combination with GOS. The most common GOS: FOS combination is 9: 1 , since it seeks to achieve a molecular weight similar to the oligosaccharides in breast milk [32]. 
Table 1. Examples of oligosaccharides present in foods and available sources.

\begin{tabular}{|c|c|c|}
\hline Type of oligosaccharide & Natural Source & Industrial Product \\
\hline Fructooligosaccharides (levans and inulin) & $\begin{array}{c}\text { Fruits and vegetables (garlic, onion, leek, } \\
\text { asparagus) }\end{array}$ & Synthesis from sucrose Hydrolysis from inulin \\
\hline Galactooligosaccharides & Beta GOS (milk) & Enzymatic \\
\hline Xylooligosaccharides & $\begin{array}{l}\text { Fruits, vegetables, honey, } \\
\text { milk }\end{array}$ & Hydrolysis from polysilanes \\
\hline Isomalto- oligosacaridos (IMOS) & Soy, honey & Hydrolysis from starch \\
\hline $\begin{array}{l}\text { Stachyose, raffinose, } \\
\text { cyclodextrins }\end{array}$ & Seed of soja & Synthesis from the starch \\
\hline
\end{tabular}

Table 2. Bacterial strains and their applications with evidence in Grades $1 a$ and $1 b$.

\begin{tabular}{|c|c|}
\hline Applications & Strains \\
\hline Acute infectious diarrhea treatment & L. rhamnosus GG, S. boulardii \\
\hline Prevention of diarrhea associated with antibiotics & L. rhamnosus GG, S. boulardii, B. Lactis, S. Thermophilus \\
\hline Functional intestinal disorders & L. rhamnosus GG, L. reuteri \\
\hline Prevention of necrotizing enterocolitis & L. acidophilus, B. bifidum, B. infantis \\
\hline Inflammatory bowel disease & VSL\#3 \\
\hline Source: Guía práctica de la Organización Mundial de gastroenterología: Probióticos y prebióticos. 2011. \\
\hline
\end{tabular}

\section{Probiotics}

Another bioactive component frequently used in infant formulas are probiotics, these can be defined as living microbial food ingredients that exert a beneficial effect on the health of the host, when administered in adequate amounts [33,34].

The main characteristic that probiotics must meet is that live microorganisms must be used in adequate quantities to obtain the desired effects. These must remain viable and active in the food during the gastrointestinal passage, to guarantee their potential beneficial effect in the host [33]. Therefore, the addition of probiotic bacteria for the preparation of functional foods depends, on the one hand, on the synergism that must be established between these crops and the fermentation initiators (ferments, starter cultures), and on the other hand, the extrinsic factors that affect or condition the viability of functional strains [27].

Among the benefits that probiotics provide to infants, are the improvement of intestinal microflora, maturation and development of humoral immunity in the first years of life $[35,36]$. They are also important for the treatment and prevention of acute infectious diarrhea in infants and children [37].

The species of microorganisms most used in infant formulas are: Bifidobacterium longum subsp. infantis, B. animalis subsp. lactis, B. longum subsp. longum, L. casei subsp. Rhamnosus and $L$. reuteri. Table 2 shows the strains that present scientific evidence [6]. The species Bifidobacterium longum subsp. Infantis has been shown to possess antirotaviral properties in in vitro and mouse studies. In addition, it has physicochemical characteristics that endorse it as a probiotic, including resistance to gastric juices, bile salts and its ability to adhere to the intestinal mucus [38,39].

\section{Symbiotics}

Symbiotic are defined as the mixing in a single product of probiotics and prebiotics that beneficially affect the host, through the implantation of live microorganisms in the gastrointestinal tract. Said implantation promotes the selective stimulation of the growth and/or activation of the metabolism of a limited number of beneficial bacteria, which produces a synergistic effect in the organism, resulting in different beneficial effects for the host health [24]. This combination enables the survival of the probiotic microorganism, since its specific substrate is readily available for fermentation. It is key to test the effect on the probiotic of the symbiotic mixture, since not all prebiotics exert the same stimulating effect of selective growth, so the prebiotic/probiotic combination has to be tested to guarantee the best possible combination.

\section{Recommendations and regulations}

The European and American Pediatrics Academies have endorsed the safety and use of probiotics in infants, emphasizing that evidence should be provided of the beneficial effects of each probiotic strain [40].

The Expert Committee of the European Society of Gastroenterology, Hepatology and Pediatric Nutrition (ESPGHAN) establishes, according to the available evidence, that the administration of formulas supplemented with probiotics to infants between 4-6 months, moderately improves the consistency and depositions by means of the contribution of Lactobacilus GG. In the case of infants older than 6 months, the possible benefits are seen on gastrointestinal infections (B. lactis), decrease in the use of antibiotics [B. lactisy, S. thermophilus, $L$. reuteri] and irritability of colic [B. lactiso, S. thermophilus] [41].

Based on research carried out, ESPGHAN concluded that the administration of probiotics in infant formulas in small infants ( $\leq$ 4 months of age) did not present clear clinical effects; although some probiotics added in infant formulas alone or in combination may have beneficial clinical effects, the routine use of formulas supplemented with probiotics for infants is not recommended $[42,43]$.

Regarding the use of prebiotics in infant feeding, no adverse effects have been found in infants born at term. However, the information currently available is not sufficient to support the widespread use of these in preterm and immunosuppressed children [44]. 
As an example, in the case of Argentina, there is no recommendation about the dose of prebiotics and prebiotics that can be added in infant formulas. Since the creation of the National Food Commission [CONAL] in 2009, progress has been made in the study of prebiotics and probiotics, resulting in the obtaining of two protocols that establish the requirements and evaluations for the inclusion of same in a food. These results were incorporated into the Argentine Food Code [CAA] at the end of 2011, through the following standards: Joint Resolution 229/2011 SPReI and 731/2011 SAGyP and Joint Resolution 261/2011 SPReI and 22/2011 SAGyP [45]. Currently the Argentine Food Code [CAA] in articles 1389 and 1390 define probiotic and prebiotic foods respectively [46].

\section{Discussion}

\section{Scientific evidences and foundations of the action of the prebiotics, probiotics and symbiotic}

In the first months of life, the microbiota of the infant fed with breast milk is fundamentally colonized by bacteria of the genus Bifidobacterium, in comparison with children fed infant formula. One of the most representative species is Bifidobacterium longum subsp. infantis, which is associated with a lower incidence of infections and a greater maturation of the immune and gastrointestinal system in children [38].

Different studies [47-49] showed that a GOS/FOS (9: 1) ratio (short chain GOS/long chain FOS) has a molecular size distribution similar to that of breast milk OSs, which favors the growth of bifidobacteria and lactobacilli; resulting in softer stools, more frequent stools, lower incidence of infectious episodes and allergic manifestations [49], as well as a lower incidence of atopic dermatitis [50-52].

In relation to the decrease in the incidence of intestinal and extraintestinal infections, it was observed that when administering formulas with prebiotics in infants, this effect was secondary to its modulating action in the intestinal microbiota, rather than its own effect [18]. Likewise, it has been indicated that the effect of milk formulas with $0.2-1.2 \mathrm{~g} / \mathrm{dl}$ of prebiotics of varied origin does not interfere in the growth of children born at term or premature $[25,53,54]$.

Clinical investigations carried out in infants [55-57] showed that the intake of infant formula supplemented with GOS/FOS at a dose of $0.8 \mathrm{~g} / \mathrm{dl}$, produces a significant increase in bifidobacteria in the feces of the children in front of the control group. In turn, it was found that this effect is dose-dependent at concentrations of 0.4 and $0.8 \mathrm{~g} / 100 \mathrm{~mL}[56,58]$.

Radke et al. evaluated the efficacy and safety of a child formula enriched with prebiotics and probiotics, on the incidence of infections during the first year of life. It was observed that the formula enriched with oligosaccharides derived from bovine milk and B. lactis was compatible with normal growth in children born at term. However, no differences were found in the reduction of the incidence of episodes of diarrhea and febrile infection in the population studied $[59,60]$.

Regarding the oral administration of Lactobacillus GG, it was shown to be effective in reducing the risk of nosocomial gastroenteritis due to rotavirus in infants [61]. In the case of the Saccharomyces boulardii strain, it has been observed that it has a beneficial effect against diarrhea associated with antibiotics [62].

The strain Lactobacillus reuteri DSM 17938 acts to improve intestinal motility, which reduces crying in infants due to infantile colic, since it has a direct effect on the nerve pathway of visceral pain. Different investigations have not observed adverse effects in the use of Lactobacillus reuteri, for the treatment of infantile colic [63].

In another study conducted in infants at 6 and 12 months of age, it was shown that children with a healthy weight had a higher number of bifidobacteria, compared to overweight children, a greater number of Staphylococcus aureus was also observed in children with obesity [64]. In relation to the use of symbiotic, a superior effect has not yet been demonstrated compared with the use of prebiotics or prebiotics independently. Currently there are few studies that have examined the impact of the use of symbiotic, associated with clinical outcomes in full-term infants fed formulas [19]. A large part of the published research has focused on the use of probiotics and prebiotics, and its relationship in the prevention of allergic diseases and food hypersensitivity in term infants [65].

A multicenter study carried out by Chua et al. evaluated the effect of a child formula with symbiotic on the composition of the intestinal microbiota in newborn infants, resulting in a reduction of intestinal enterobacteria, as a result of the acidic intestinal environment generated by the symbiotic modulation of the intestinal microbiota and the increase of acetate [66]. Regarding the evaluation of infant growth patterns (weight gain, length and cephalic perimeter), no significant scientific findings have been reported that support the addition of symbiotic in the milk formula. In relation to stools, it has been observed that symbiotic significantly increase the frequency of bowel movements. Not enough evidence was found to affirm that symbiotic reduce the risk of infections or reduce the use of antibiotics. Therefore, it is suggested to continue research to support the routine supplementation of infant formulas with symbiotic, prebiotics or prebiotics [27].

\section{Conclusion}

Currently, there is no scientific evidence to suggest that the addition of probiotics in formulas for infants can be harmful in children born at term and without health problems. Evidence about clinical efficacy through the addition of prebiotics is not enough to recommend routine use in infant formulas. In relation to the use of specific prebiotics, mainly oligosaccharides, it has been possible to obtain evidence of their beneficial effects. There is evidence that the manipulation of the gut microbiota with foods containing pre and probiotics, contribute to a possible health benefit if the initial flora is abnormal. The researchers analyzed did not find available data to establish if symbiotic are superior to probiotics or prebiotics. Therefore, supplementation with symbiotic is not routinely supported in infant formulas for full-term infants. There is not enough evidence to recommend supplementation with probiotics or prebiotics of milk formulas intended for preterm infants, because no better growth has been proven. It is necessary to emphasize that the functional food market is in development and the mechanisms of action of the components are better known every day. However, in 
relation to the consumption of probiotics, new research with scientific support is needed, in order to make recommendations, and establish with specificity the types of strains, species and consumption doses that are beneficial for health.

\section{References}

1. Gonzalez Garcia MI, Ibanez Martin R. Know, believe and buy the role of scientific knowledge in attitudes towards functional foods and the environment. Dialnet 2008;20(1):115-133.

2. Fuentes-Berrio L. Functional foods: Impact and challenges for the development and welfare of Colombian society. Biotechnology in the Agricultural and Agroindustrial Sector. 2015;13(2):140-149.

3. Duran CR, Valenzuela BA. The Japanese experience with FOSHU foods: the true functional foods? Rev Chil Nutr 2010;37(2):224-233.

4. Arias LD, Montano LN, Velasco MA. Functional foods: application advances in agro-industry. Tecnura, Francisco José de Caldas District University. 2018;57(22):55-68.

5. Palou A, Serra F. European perspectives on functional foods. Food, Nutrition and Health. Dialnet. 2000;7(3):76-90.

6. Almagro MC, Moreno JA, Jimenez LJ, et al. New ingredients in infant formulas: health and functional benefits. Nutr. Hosp. 2017;34(4):8-12.

7. Richard C, Lewis ED, Field CJ. Evidence of the essentiality of arachidonic acid and docosahexaenoic acid in the maternal and infant postnatal diet for the development of the baby's immune system early in life. Canadian Science Publishing. 2016;41(5):461-475.

8. Veereman-Wauters G. Intestinal Microbiota and Health in Childhood. Universitair Kinderziekenhuis Brussel, Vrije Universiteit Brussel. 2011;30(4):111-117.

9. Moles L, Gueimonde M, Rodríguez JM. Influence of perinatal microbiomes in premature birth and health of premature infants: Influencing factors and modulation strategies. J Pediatr Gastroenterol Nutr. 2016;63(6):193-200.

10. Ministry of the Presidency of Spain. Approval of specific technical-sanitary regulations for infant formulas and follow-on formulas. State official newsletter. Consolidated legislation. Royal Decree 867.2008.

11. Ministry of Health. National Direction of Maternity, Childhood and Adolescence. Situation of breastfeeding in Argentina. Year 2015.

12. Minana IV. Functional foods in pediatrics. Spanish Association of Pediatrics of Primary Attention. Child Nutrition Section. Hospital of Xàtiva, Valencia. 2006.

13. Zhong H. Impact of early events and lifestyle on the gut microbiota and metabolic phenotypes in young school-age children. Microbiome. 2019;7(2):2-14.

14. Sevilla Soldan PR, Zalles Cueto L, Santa Cruz Gallardo W. Breastfeeding vs. New artificial dairy formulas: Impact evaluation in development, immunity, body composition in mother/child pair. Gac Med Bol. 2011;34(1):6-10.
15. Quintaes K, Barbera D, Reyes AA. Phospholipids in breast milk and infant formulas: Benefits and needs for proper nutrition of children. Crit Rev Food Sci Nutr. 2016;56(11):1880-92.

16. Plaza Diaz J, Fontana L, Gil A. Oligosaccharides of human milk and the development of the immune system. Nutrients. 2018;10(8):43-52.

17. Chirdo FG. Prebiotics in children's health. Argentine Archives of Pediatrics. 2011;109(1):49-55.

18. Klopp A. Modalities of infant feeding and the risk of childhood asthma: a prospective study of birth cohort. Pediatrics Magazine. 2017;190(1):192.

19. Diplock AT. Scientific concepts of functional foods in Europe: Consensus document. Brit J Nutr. 1999;81:S1-S27.

20. Li R. Breastfeeding and risk of infection at 6 years. Pediatrics. 2014;134(1):13-20.

21. Mousavi KA, Fakhri Y. Probiotics and prebiotics as functional foods: state of the art. Current Nutrition \& Food Science. 2019;15(1):33-40.

22. Komatsu TR, Buriti Flavia CA, Saad SM. Innovation, persistence and creativity overcoming barriers in the development of probiotic foods. Rev Bras Cienc Farm. 2008;44(3):329-347.

23. Lopez-Marin BE, Alvarez-Rivera JM, Carvajal de Pabon LM. Development of two infant formulas as an economical and healthy alternative for food and nutritional security of the nursing population. Univ Health. 2016;18(2):291-301.

24. Gibson GR. Fibre and effects on probiotics(The prebiotic concept). Clin Nutr Suplements. 2004;(Suppl 1):25-31.

25. Olagnero G. Functional foods: Fiber, prebiotic, probiotic and symbiotic. DIAETA. 2007;121(25):20-33.

26. Castaneda GC. Updating in prebiotics. Rev Cubana Pediatr. 2018;90(4):e648.

27. Mugambi MN, Musekiwa A, Lombard M, et al. Probiotics, prebiotics infant formula use in preterm or low birth weight infants: A systematic review. Nutrition Journal. 2012;11:58.

28. Yu LC, Wang JT, Wei SC, et al. Host-microbial interactions and regulation of intestinal epithelial barrier function: From physiology to pathology. World J Gastrointest Pathophysiol. 2012;3(1):27- 43.

29. Shahramian I. The effects of prebiotic supplementation on weight gain, diarrhea, constipation, fever and respiratory tract infections in the first year of life. J Paediatr Children's Health. 2018;54(8):875-880.

30. Moro G, Minoli I, Mosca M, et al. Dosage-related bifidogenic effects of galacto and fructooligosaccharide in formula-fed term infants. J Pediatr Gastroenterol Nutr. 2002;34(3): 291- 295.

31. Lim SM, Kim DH. Bifidobacterium adolescentis IM38 mejora la colitis inducida por la dieta rica en grasas en ratones al inhibir la activación de NF- $\kappa \mathrm{B}$ y la producción de lipopolisacáridos por microbiota intestinal. Investigación de nutrición. 2017;41(1):86-96. 
32. Brunser TO. The role of bifidobacteria in the functioning of the human organism. Rev. Chil. Nutr. 2013;40(3):303-308.

33. Araya LH, Lutz RM. Functional and healthy foods. Rev. Chil. Nutr. 2003;30(1):8-14.

34. Bird AR. Resistant starch, fermentation of the large intestine and a broader perspective of prebiotics and probiotics. beneficial microbes. 2019;4(1):423-431.

35. McGuirre MK, McGuirre MA. Human milk: prototypical probiotic food of mother nature? Adv Nutr. 2015;6(1):112123.

36. Lemaire M, Le Huerou-Luron I, Blat S. Effects of the composition of infant formula on long-term metabolic health. J Dev Orig Health Dis. 2018;9(6):573-589.

37. Munoz JA, Chenoll E, Casinos B, et al. Novel prebiotic Bifidobacterium longum subsp. Infantis CECT 7210 strain active against rotavirus infections. Appl Environ Microbiol. 2011;77(24):8775-83.

38. Bozensky J, Hill M, Zelenka R, et al. Prebiotics Do Not Influence the Severity of Atopic Dermatitis in Infants: A Randomised Controlled Trial. PLoS ONE. 2015;10(11):e0142897.

39. Escribano J. Bifidobacterium longum subsp infantis CECT7210-supplemented formula reduces diarrhea in healthy infants: A randomized controlled trial. Pediatric Research. 2018;83(6):1120-1128.

40. Alvarez-Calatayud G, Perez-Moreno J, Tolin M, et al. Aplicaciones clínicas del empleo de probióticos en pediatría. Nutr. Hosp. 2013;28(3):564-574.

41. Moreno C. A complex issue. Regulations and functional foods. Secretariat of Agriculture, Livestock and Fishing. Argentine Food. 2016.

42. Szajewska A, Guarino I, Hojsak F, et al. Use of probiotics for management of acute gastroenteritis: A position paper by the ESPGHAN Working Group for Probiotics and Prebiotics. J Pediatr Gastroenterol Nutr. 2014; 54:531-539.

43. Olveira G, González-Molero I. Updating of probiotics, prebiotics and symbiotics in clinical nutrition. Endocrinology and Nutrition 2016;63(9):437-508.

44. Montse Vilaplana B. Probiotics and health. Elsevier 2015;29(3):5-50.

45. Argentine Food Code. Chapter XVII "Regimen or dietetic foods". Artícle 1389(Res. Conj. SPReI N ${ }^{\circ}$ 261/2011 y SAGyP N $\left.{ }^{\circ} 22 / 2011\right)$.

46. Argentine Food Code. Chapter XVII "Regimen or dietetic foods". Article 1385(Res. Conj. SPReI N95/2008 y SAGPyA N 358/2008).

47. Mao B. In vitro fermentation of fructooligosaccharides with human gut bacteria. Food Funct. 2015;6(3):947-954.

48. Lingfen X. A double-blind randomized trial on growth and food tolerance with Saccharomyces boulardii CNCM I-745 in preterm infants fed formula. Journal Pediatrics. 2016;92(3):296-301.
49. Sherman PM, Cabana M, Gibson GR, et al. Potential roles and clinical utility of prebiotics in newborns, infants, and children: proceedings from a global prebiotic summit meeting, New York City, June 27-28, 2008. J Pediatr. 2009;155(5):61-70.

50. Roze JC. An $\alpha$-lactalbumin-enriched and symbiotic-supplemented v. a standard infant formula: a multicentre, doubleblind, randomised trial. Br J Nutr. 2012;107(11):1616-22.

51. Narayanan R, Subramonian B. Effect of prebiotics on bifidobacterial species isolated from childhood feces. Indian Magazine of Traditional Knowledge. 2015;14(2):285-289.

52. Deshpandey AK, Ryan CA, Dempsey EM, et al. The neonatal gut harbours distinct bifidobacterial strains. Archives of Childhood Illness. 2015;100(5):405-410.

53. Wopereis H. Intestinal microbiota in infants at high risk for allergy: Effects of prebiotics and role in eczema development. J Allergy Clin Immunol. 2018;141(4):1334-1342.

54. Butel MJ, Waligora-Dupriet AJ, Wydau-Dematteis S. The developing gut microbiota and its consequences for health. J Dev Orig Health Dis. 2018;9(6):590-597.

55. Baranowski JR, Claud EC. Necrotizing enterocolitis and the preterm infant microbiome. Adv Exp Med Biol. 2019;1: $1-12$.

56. Vandenplas Y, Zakharova I, Dmitrieva Y. Oligosaccharides in infant formula: more evidence to validate the role of prebiotics. Br J Nutr. 2015;113(9):1339-44.

57. Ringel-Kulka T, Kotch JB, Jensen ET, et al. Randomized, double-blind, placebo- controlled study of synbiotic yogurt effect on the health of children. J Pediatr. 2015;166(6):14751481.

58. Kent RM, Fitzgerald GF, Hill C, et al. Novel approaches to improve the intrinsic microbiological safety of powdered infant milk formula. Nutrients. 2015;7(2):1217-44.

59. Simeoni U. Intestinal microbiota analysis reveals a marked change towards bifidobacteria by an infant formula that contains a symbiotic oligosaccharide derived from bovine milk and B ifidobacterium animalis subsp. Lactis CNCM I-3446. Environmental Microbiology. 2016;18(7):2185-2195.

60. Radke M. Starter formula enriched in prebiotics and probiotics ensures normal growth of infants and promotes gut health: A randomized clinical trial. Pediatr Res. 2017;81(4):622-631.

61. Rao S, Srinivasjois R, Patole S. Prebiotic supplementation in full-term neonates: a systematic review of randomized controlled trials. Arch Pediatr Adolesc Med 2009;163(8):755764.

62. Can M, Besirbellioglu BA, Avci IY, et al. Prophylactic Saccharomyces boulardii in the prevention of antibioticassociated diarrhea: a prospective study. Med Sci Monit. $2006 ; 12: 19-22$.

63. Savino F, Cordisco L. Lactobacillus reuteri DSM Infantile Colic: A randomized, double- blind placebo-controlled trial. Pediatrics. 2010;126(3):e526-33. 
64. Kalliomaki M, Collado MC, Salminem S, et al. Early differences in fecal microbiota composition in children may predict overweight. Am J Clin Nutr. 2008;87(3):534-538.

65. Osborn DA. Sinn JKH. Prebiotics in infants for prevention of allergic disease and food hypersensitivity. Cochrane Database of Systematic Reviews: Reviews. 2007.

66. Szajewska H. Effects of infant formula supplemented with prebiotics compared with synbiotics on growth up to the age of 12 months: A randomized controlled trial. Pediatr Res. 2017;81(5):752-758

\section{*Correspondence to:}

\section{Federik M}

National University of Lanus

Department of collective health

Climbing Remedies, Province of Buenos Aires, Argentina.

Tel: +54 1151750861

Email: marianelaafederik@gmail.com 